\title{
The Technique and Cost of Radiosurgery for the Treatment of 1-3 Brain Metastases
}

\author{
Waseem Sharieff, Anthony Whitton, Tom Chow, Doug Wyman, James Wright, \\ Jeffrey N. Greenspoon
}

\begin{abstract}
Background: Radiosurgery can be delivered through a variety of modalities including robotic and fixed gantry Linacbased systems. They appear equally effective and safe. Thus, community need and costs remain the main determinants for choosing a given modality. We performed an economic evaluation to identify settings in which one modality could be preferred over the other. Methods: Using local estimates of resource volumes and unit prices, we computed the incremental cost/patient of robotic radiosurgery compared to fixed-gantry radiosurgery from a payer's perspective. By varying parameters of resource volumes, we performed a probabilistic analysis stratified by number of brain lesions. In addition, we performed sensitivity analyses to examine the effect of patient volume on cost/patient. Results: The cost of robotic radiosurgery was $\$ 4,783 /$ patient, and cost of fixed-gantry radiosurgery was $\$ 5,166 /$ patient. The mean incremental cost was \$-383 (95\% interval: \$-670, \$110) for all lesions, \$78 (\$23, \$123) for solitary lesions, and \$-610 (\$-679, \$-534) for multiple lesions. The cost/patient of robotic radiosurgery varied from $\$ 5,656$ (low volume setting) to $\$ 4,492$ (high volume setting). Conclusion: In settings of moderate to high volume (6-10 hours of daily operation), and in multiple lesions, robotic radiosurgery is more cost effective than fixed-gantry radiosurgery.
\end{abstract}

RÉSUMÉ: Technique utilisée et coût de la radiochirurgie pour le traitement de 1 à 3 métastases cérébrales. Contexte : La radiochirurgie peut être administrée au moyen de différentes modalités dont les systèmes robotisés et de type LINAC à portique fixe. Ces modalités de traitement semblent être également efficaces et sûres. Le choix de l'une ou l'autre semble déterminé par les besoins de la communauté et les coûts. Nous avons effectué une évaluation économique pour identifier le contexte dans lequel l'une pourrait être préférée à l'autre. Méthode : Nous avons calculé ce que coûterait par patient la radiochirurgie robotisée comparée à la radiochirurgie à portique fixe, du point de vue du payeur, au moyen des estimés locaux du volume des ressources et des prix unitaires. En variant les paramètres du volume des ressources, nous avons effectué une analyse probabiliste stratifiée selon le nombre de lésions cérébrales à traiter. De plus, nous avons effectué des analyses de sensibilité pour examiner l'effet du volume de patients sur le coût par patient. Résultats : Le coût de la radiochirurgie robotisée était de $4783 \$$ par patient et le coût de la radiochirurgie à portique fixe était de $5166 \$$ par patient. Le coût additionnel moyen était de $-383 \$$ (intervalle de confiance à $95 \%$ : $-670 \$$ à $110 \$$ ) pour toutes les lésions, $78 \$(23 \$$ à $123 \$$ ) pour les lésions uniques et $-610 \$(-679 \$$ à $-534 \$)$ pour les lésions multiples. Le coût par patient de la radiochirurgie robotisée variait de $5656 \$$ (si le volume était faible) à 4 492\$ (si le volume était élevé). Conclusion : Si le volume est de modéré à élevé et en opération de 6 à 10 heures par jour, la radiochirurgie robotisée est plus avantageuse au point de vue économique que la radiochirurgie à portique fixe pour traiter les lésions multiples.

Can J Neurol Sci. 2013; 40: 795-799

Brain metastases occur in up to $40 \%$ of patients with cancer. $^{1,2}$ Randomized trials have demonstrated improved survival and quality of life when radiosurgery is added to conventional whole brain radiation therapy (WBRT). ${ }^{3}$ Radiosurgery has thus become the standard of care in conjunction with WBRT for the treatment of brain metastases. Currently, its largest use is in the treatment of brain metastases. ${ }^{4}$

Radiosurgery can be delivered by either modifying an existing linear accelerator 'fixed-gantry' system (which can also deliver 'other' radiotherapy treatments), or by acquiring a dedicated radiosurgery unit, such as a 'robotic' system. ${ }^{5}$ Both methods produce accuracy to within $1 \mathrm{~mm}$ of target volume, and thus both are deemed equally effective and equally safe. ${ }^{6-8}$ However, community need, costs, patient preferences and budget constraints are important considerations in decisionmaking. We performed an economic evaluation of robotic radiosurgery for brain metastases in comparison to fixed gantry radiosurgery in order to help guide decision makers.

\section{MeTHODS \\ Population}

At Juravinski Cancer Centre (JCC) in 2012, 550 courses of radiosurgery were delivered to a total of 325 patients with one to three brain metastases. ${ }^{9}$ Of these, $45 \%$ had a solitary lesion, $39 \%$ had two lesions and $16 \%$ had three lesions. On average, a patient received 1.7 courses of radiosurgery. We used these parameters to simulate a cohort of 5000 male and female adult patients.

From the Department of Radiation Oncology (WS, AW, TC, DW, JW, JNG), Juravinski Cancer Centre, Hamilton Health Sciences; Department of Oncology (WS, AW, TC, DW, JW, JNG), McMaster University, Hamilton Ontario; Department of Radiation Oncology (WS), Cape Breton Regional Cancer Centre, Sydney; Department of Radiation Oncology (WS), Dalhousie University, Halifax, Nova Scotia.

Received April 24, 2013. Final Revisions Submitted June 18, 2013. Correspondence to: Waseem Sharieff, Department of Radiation Oncology, Cape Breton Regional Cancer Centre, Sydney, Nova Scotia, B1P 1P3, Canada.

Email: doc.sharieff@utoronto.ca. 


\section{Comparisons}

We compared two radiosurgery modalities: (a) Robotic radiosurgery; and (b) Fixed-gantry radiosurgery. We compared resource volumes including planning and treatment times in patients who received Robotic radiosurgery to patients who received Fixed-gantry radiosurgery.

\section{Radiosurgery}

Radiosurgery refers to stereotactically guided conformal irradiation of a defined target volume in a single session; when delivered in two to five sessions, the procedure is called fractionated radiosurgery. Stereotaxis refers to a precise threedimensional (3D) mapping technique to guide a procedure.

\section{Robotic radiosurgery}

At JCC, Cyberknife $\AA$ is used for robotic radiosurgery. Radiation therapists position the patient supine and immobilize the head with a thermoplastic face mask. Next, they perform a CT simulation with slice thickness in the range of $1.0-1.25 \mathrm{~mm}$. After the images are exported to the planning software, a dosimetrist performs a fusion between simulation computed tomogram (CT) and a gadolinium enhanced T1 weighted magnetic resonance imaging (MRI) (slice thickness of $1 \mathrm{~mm}$ ) obtained within two weeks of expected treatment date. The dosimetrist also sets up a reference point called 'alignment centre' on the simulation CT (Figure 1A). The radiation

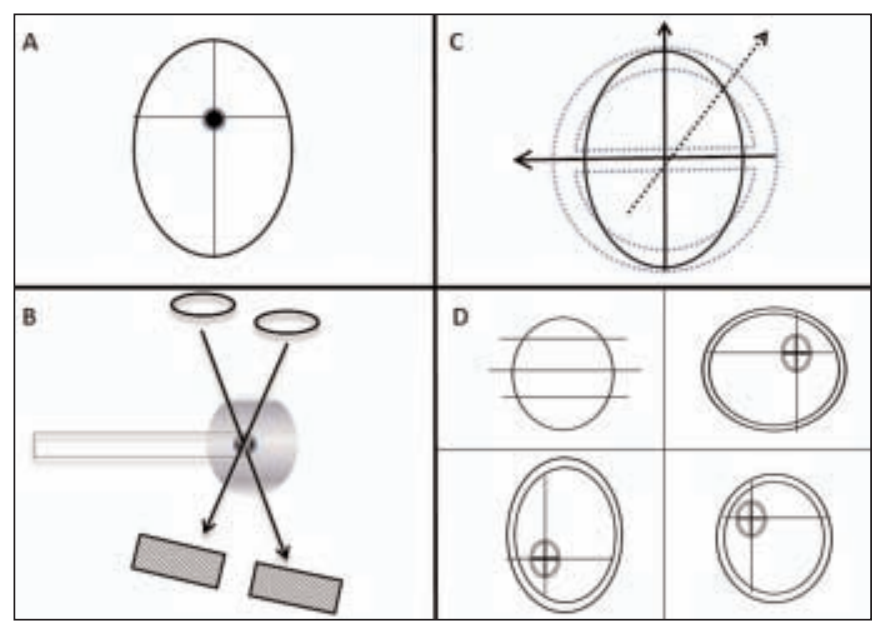

Figure 1: Stereotactic radiosurgery. Panel A shows the alignment centre on an axial plane for a robotic radiosurgery plan. Panel B schematically shows a robotic radiosurgery unit. The ovals are the cameras located on the ceiling, arrows represent the $K V$ beams intersecting at the imaging center, rectangles at the bottom are the image detectors located on the floor, rectangle at the center represents the couch on which the patient is positioned. Note that the alignment center at the head end of the couch is aligned to the imaging center which provides the external $3 D$ coordinates. Panels $C \& D$ diagrammatically show fixed-gantry radiosurgery. Panel $C$ shows the head (solid) in a stereotactic head frame (dotted) that provides the external 3D coordinates (arrows). Panel $D$ shows various cut levels in a cone beam CT scan (upper left); upper right and bottom sub-panels show overlay of cone beam CT on simulation CT in all 3 planes. Note that the lesion shown on simulation $C T$ is the area of interest which is matched to bony landmarks on cone beam CT in all 3 planes. The same process is repeated for each lesion. oncologist and the neurosurgeon delineate the clinical target volumes (CTVs). Clinical target volumes comprises of the gross lesion including its enhancing component. The dosimetrist geometrically expands each CTV by $1 \mathrm{~mm}$ to planning target volume (PTV). If the CTV abutts a critical organ (e.g., brain stem), no PTV expansion is given at the interface between CTV and the critical organ. The radiation oncologist prescribes the dose to the PTV which depends on the maximal dimension. The dosimetrist generates a plan in which 50-200 beams from various directions and path lengths traverse the PTV. The dose is prescribed to the isodose line that covers $95 \%$ of the PTV, which is typically $65-85 \%$ isodose line (when the plan is normalized to maximum point dose). This results in $>100 \%$ of the prescription dose in the centre of the PTV, and in a steep dose gradient outside the PTV wherein the dose rapidly falls off. The physicist reviews the plan and the radiation oncologist approves it. The planning system generates two sets of digitally reconstructed radiographs (DRRs) for alignment and lesion tracking.

At the time of treatment delivery, therapists position and immobilize the patient as before. The alignment centre on the reference DRRs is aligned to the imaging centre (Figure 1B). The imaging centre provides the $3 \mathrm{D}$ coordinates for stereotaxis. It is a point in space in the treatment unit which is intersected by two orthogonal kilovoltage beams from two cameras located on the ceiling. Two image detectors located on the opposite side of the floor detect these beams. These cameras continuously take images in real time which are matched to the reference DRRs for target localization. The robot on which the linear accelerator is mounted corrects for any misalignment at the time of set up and during the course of treatment delivery.

\section{Fixed-gantry based radiosurgery}

At JCC, Varian Trilogy ${ }^{\circledR}$ linac with brain lab radiosurgery hardware and software is used. The therapists position the patient supine and immobilize patient's head in a re-locatable stereotactic frame that provides the $3 \mathrm{D}$ co-ordinates for stereotaxis (Figure 1C). The rest of the planning is similar except that arcs and multiple isocenters are used for each target.

At the time of treatment delivery, the therapists set up the patient as before. They use a cone beam CT scanner which is attached to the linear accelerator to acquire set-up images. They match the images by fusion to the planning CT overlaying them (Figure 1D). The radiation oncologist reviews the images for an acceptable match. If unacceptable, the therapists manually shift and or rotate the head frame to correct the misalignment. They re-acquire the cone beam CT images for matching until an acceptable match is obtained. The linear accelerator delivers radiation to the lesion. Unlike the CyberKnife, the residual errors are not corrected, but are kept below $1 \mathrm{~mm}$ and 1 degree. The therapists repeat the whole process for the next lesion or isocenter. Fixed gantry treatments are planned and delivered using fixed cones or dynamic conformal multi-leaf collimators in four to six arcs.

\section{Type of evaluation and perspective}

We carried out economic evaluation of treating one to three brain metastases patients with Robotic radiosurgery from a payer's perspective. This involved calculating incremental cost per patient and the total budget impact. 
Table 1: Resource volumes

\begin{tabular}{|c|c|c|}
\hline & $\begin{array}{l}\text { Robotic } \\
\text { Radiosurgery }\end{array}$ & $\begin{array}{l}\text { Fixed Gantry } \\
\text { Radiosurgery }\end{array}$ \\
\hline \multicolumn{3}{|l|}{$\begin{array}{l}\text { Resource volumes } \\
\text { Clinic visit }^{1}\end{array}$} \\
\hline Radiation Oncologist & 1 & 1 \\
\hline Neurosurgeon & 1 & 1 \\
\hline Nurse & 1 & 1 \\
\hline Consultation time $\mathrm{min} /$ patient & 60 & 60 \\
\hline Review time min/patient & 10 & 10 \\
\hline Follow up time $\mathrm{min} /$ patient & 30 & 30 \\
\hline \multicolumn{3}{|l|}{ Simulation $^{2}$} \\
\hline Radiation Therapist(s) & 3 & 3 \\
\hline Time min/patient & 2 & 10 \\
\hline \multicolumn{3}{|l|}{ Planning ${ }^{3}$} \\
\hline Radiation Oncologist & 1 & 1 \\
\hline Physicist & 1 & 1 \\
\hline Time min/patient & 60 & 60 \\
\hline Dosimetrist & 1 & 1 \\
\hline Time min/patient & 360 & 360 \\
\hline \multicolumn{3}{|l|}{ Delivery $^{4}$} \\
\hline Physicist & 1 & 1 \\
\hline Radiation Therapist(s) & 3 & 3 \\
\hline Time min/patient & 60 & $90-180$ \\
\hline \multicolumn{3}{|l|}{ QA } \\
\hline Physicist & 1 & 1 \\
\hline Time hr/year & 608 & 509 \\
\hline
\end{tabular}

${ }^{1}$ one consultation, one review, and one follow up visit; ${ }^{2}$ immobilization and CT simulation; ${ }^{3}$ target volume and organ at risk delineation, beam arrangement, dose computation, revisions, and DRR generation; ${ }^{4}$ set up and alignment, target tracking and treatment, image verification.

\section{The model}

The starting point of the model was a cohort of patients assigned to Robotic radiosurgery or to Fixed-gantry radiosurgery. The patients underwent initial clinical assessment, followed up treatment planning and delivery. Thereupon, they were seen in review clinic and once again in follow-up. They were then discharged to be followed by the referring physician. The time span of the model was one year.

\section{Assumptions}

We assumed that: (1) both radiosurgery modalities were equally effective and equally safe; (2) all patients consented for the treatment, and remained stable during the planning, treatment and follow-up; (3) fixed-gantry system operated for ten hours daily, five days a week and it treated other diseases besides brain metastases, but robotic system operated for six hours daily, five days a week and it treated a limited number of other diseases; and (4) each system was acquired through a bank loan for ten years.

\section{Data sources}

For population characteristics, and resource volumes, we used local estimates. For unit prices, we used local purchase data, and Ontario Health Insurance (OHIP) billing codes, ${ }^{10}$ where applicable; for long term investment items (e.g., machines), we used 5\% interest rate and ten year amortization period to calculate annual payments. Table 1 shows resource volumes.

\section{Resource volumes}

Using means, standard deviations, and minimum-maximum values for continuous data, and proportions for dichotomous data, we used Monte Carlo simulation to generate resource volumes of planning and treatment times stratified by solitary versus multiple brain metastases. A newly launched study provided planning and treatment times. ${ }^{11}$

\section{Costs}

To capture all costs, i.e., those of the equipment plus those related to its administration and maintenance, and other costs related to clinical assessment, treatment planning, delivery and follow up, we included cost items as machine, renovation, warranty, immobilization device, physician fees, salaries of nurses, therapists, and physicists (Table 2). We did not include costs related to loss of productivity from hospitalization, disability or death; we excluded patients' out-of-pocket

Table 2: Costs

\begin{tabular}{lll}
\hline & Robotic Radiosurgery & Fixed Gantry Radiosurgery \\
\hline Unit Price & & $\$ 152.40$ \\
Radiation Oncologist fee/consult & $\$ 152.40$ & $\$ 121.10$ \\
Neurosurgeon fee/consult & $\$ 121.10$ & $\$ 37.05$ \\
Radiation Oncologist fee/review & $\$ 37.05$ & $\$ 68.90$ \\
Radiation Oncologist fee/follow up & $\$ 68.90$ & $\$ 58.25$ \\
Neurosurgeon fee/follow up & $\$ 58.25$ & $\$ 811.15$ \\
Radiation Oncologist fee/plan & $\$ 811.15$ & $\$ 538.40$ \\
Neurosurgeon fee/plan & $\$ 538.40$ & $\$ 52.00$ \\
Radiation Therapist salary/hr & $\$ 52.00$ & $\$ 52.00$ \\
Dosimetrist salary/hr & $\$ 52.00$ & $\$ 94.00$ \\
Physicist salary/hr & $\$ 94.00$ & $\$ 50$ \\
Nurse salary/hr & $\$ 50$ & $\$ 180$ \\
Immobilization device & $\$ 35$ & $\$ 1,000$ \\
MRI/plan & $\$ 1,000$ & $\$ 476,235.84$ \\
Overhead & & $\$ 8,580$ \\
Annual payment machine + installation & $\$ 622,191.84$ & $\$ 14,400$ \\
Annual Maintenance & & \\
\hline
\end{tabular}

${ }^{1}$ Based on $5 \%$ interest rate and 10 year amortization period 
expenses. Finally, we multiplied the unit prices of cost items with resource volumes to compute the total costs for the two groups.

\section{Outcomes}

The main outcome was cost per patient related to each modality. We did not include overall survival, quality adjust life years, or toxicity because there are no data to suggest that one modality is better than the other for any of these outcomes. ${ }^{3}$

\section{Analysis}

First, we calculated cost per patient for both groups. Next, we subtracted cost per patient of robotic group from that of fixedgantry group. This yielded the incremental cost/patient attributable to robotic radiosurgery. Since time span was one year, we did not apply any discounting to costs.

To account for the uncertainty in daily hours of operation, we performed a sensitivity analysis by varying this parameter from two hours (low volume setting) to ten hours (high volume setting). To account for uncertainty in percentage of patients with multiple lesions out of the total patients, we varied this parameter from $0 \%$ to $100 \%$. In addition, we performed a probabilistic analysis by using Monte Carlo simulations on treatment times and number of lesions, and computed $95 \%$ intervals on the incremental cost/patient.

For budget impact, we took the difference of annual cost of acquiring the unit (purchase and installment) and its maintenance, between robotic and fixed-gantry systems. We assumed a variation of $\pm 5 \%$ in purchase price, and up to $15 \%$ increment in repair costs depending upon utilization.

We reported costs in Canadian dollars (one Canadian dollar $(C A D)=1.01$ United States of America (US) dollar; Dec 2012).

\section{RESULTS}

Both groups had similar patient characteristics. In each group, $45 \%$ had solitary metastasis, $39 \%$ had two and $16 \%$ had three metastases. Treatment planning time was approximately six hours in each group. However, treatment delivery time was $60 \pm$ 5 minutes in the robotic group, and $140 \pm 45$ minutes in the fixed-gantry group.

The cost of radiosurgery was $\$ 4,783 /$ patient for robotic and $\$ 5,166 /$ patient for fixed-gantry system. In sensitivity analyses, the cost of robotic radiosurgery varied from $\$ 5,656 /$ patient in low volume setting to $\$ 4,492 /$ patient in high volume setting.

When the percentage of patients with multiple lesions out of the total patients, was varied from $0 \%$ to $100 \%$, the cost of fixedgantry radiosurgery was higher than the cost of robotic radiosurgery except when $<10 \%$ patients had multiple lesions (Figure 2).

The incremental cost/patient was \$-383 (95\% interval: \$-670, $\$ 110)$ for all lesions, $\$ 78(\$ 23, \$ 123)$ for solitary lesions, and \$610 (\$-679, \$-534) for multiple lesions. The annual budget impact was $\$ 151,776(\$ 144,478, \$ 159,947)$.

\section{DISCUSSION}

We compared the cost effectiveness of robotic radiosurgery to fixed gantry radiosurgery for the treatment of one to three brain metastases. When total investment costs were considered,

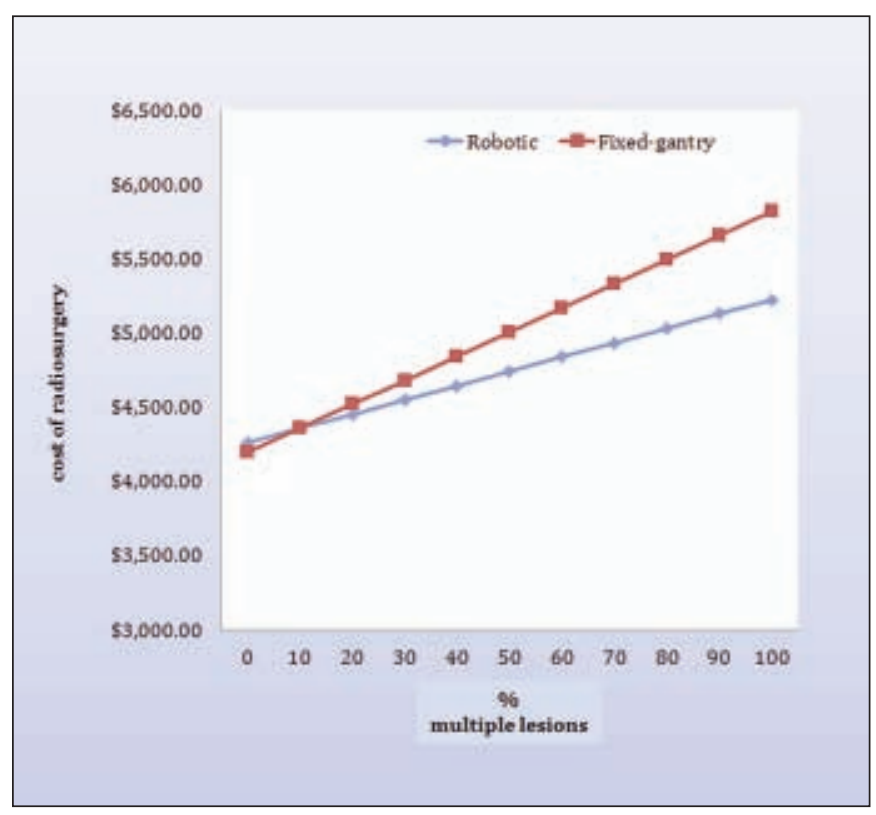

Figure 2: Cost of radiosurgery. Sensitivity analysis showing robotic (blue) and fixed-gantry (red) radiosurgery costs in relation to the percentage of patients with multiple lesions. Note that except when $<$ $10 \%$ patients have multiple lesions (i.e., $>90 \%$ patients have solitary lesions), the cost of fixed-gantry radiosurgery is higher than that of the robotic radiosurgery.

robotic radiosurgery was more costly than fixed-gantry-based radiosurgery. When cost per patient was considered, robotic radiosurgery was more cost effective than fixed-gantry-based radiosurgery. When number of lesions were considered (single versus multiple), cost per patient was lower for fixed-gantry radiosurgery for solitary lesions, compared to robotic radiosurgery. This was related to longer treatment times associated with multiple lesions for fixed-gantry radiosurgery.

There is limited literature on the cost effectiveness of radiation therapy for brain metastases. Of the studies in US, Lal and colleagues estimated that the cost of radiosurgery plus whole brain radiation was $\$ 74,000$, and the cost of radiosurgery alone was $\$ 119,000$ (US\$). ${ }^{12}$ In their study, radiosurgery was delivered by a fixed-gantry system to patients with Recursive Partition Analysis (RPA) class 1 or 2 and one to three brain metastases. From the perspective of a tertiary care cancer centre, they collected costs of the initial treatment as well as subsequent treatments (i.e., for local recurrence with radiation/surgery) up to death or end of study period (median follow-up 9.5 months). We limited our cost analysis from the time of referral to a radiation oncologist to one year post treatment. Thus, our estimate of radiosurgery cost $(\$ 5,166)$ is relatively low. However, our results are similar to Mehta and colleagues. ${ }^{13}$ They used their local hospital's cost-accounting system to generate costs - the cost of whole brain radiation was $\$ 6,500$ and the cost of whole brain radiation plus radiosurgery was $\$ 15,102$ (US\$).

In a European study, Vuong and colleagues estimated the cost of initial radiosurgery with Gamma knife and subsequent local treatments to be $€ 9,964$ (\$12,998 CAD). ${ }^{14}$ Their costing was based on a payer's perspective from time of initial treatment to 
death or 5.5 years of follow-up. They excluded costs of followup visits, and adjunct treatments (chemotherapy, palliative, rehabilitation). When the differences in costing methods and study setting, are taken into account, their results appear similar to ours. Nevertheless, we have described the cost items and resource volumes so that by using local unit prices, decision makers could generate cost estimates in their own settings.

Our results are sensitive to patient volume. This finding is in agreement with the cost analysis of Königsmaier and colleagues. ${ }^{15}$ They compared cost of radiosurgery with Gamma knife in comparison with a fixed-gantry system. The cost of Gamma knife was DM 13,106 in low volume setting and DM 4,002 in high volume setting; the corresponding cost of fixedgantry radiosurgery was DM 13,616 and DM 4,328. Thus, cancer centres that do not have enough patients to use the robotic system for six to eight hours daily may choose to commission their fixed gantry units for stereotactic application, rather than investing in a dedicated robotic system.

Our results should be used in the context of study limitations. First, we performed costing from the payer's perspective. Thus, out-of-pocket patient costs associated with commuting and parking were not captured. Since, robotic radiosurgery is associated with shorter treatment time, the inclusion of these costs would further favor robotic over fixed-gantry radiosurgery. Second, the longer treatment time associated with fixed-gantry radiosurgery was due to manual shifts and rotation of the couch to achieve an image match. A robotic couch is commercially available for fixed-gantry radiosurgery that may allow faster treatment delivery times compared to a manual couch. However, when the price of a robotic couch is added to the price of fixedgantry system, the total price equals that of a robotic radiosurgery system. Finally, we did not perform cost utility analyses. There are no data to suggest a difference in survival or quality of life with the use of robotic radiosurgery compared to fixed-gantry radiosurgery. On the contrary, a subgroup analysis of RTOG 9508 showed that dedicated radiosurgery (Gamma knife $\left.{ }^{\circledR}\right)$ and fixed gantry based radiosurgery were equivalent when survival was compared. ${ }^{3}$ Thus, we do not believe that omission of these analyses would alter our conclusions. Nevertheless, this is under investigation. ${ }^{11}$

\section{Conclusions}

In settings of moderate to high volume (six to ten hours of daily operation), and in multiple lesions, robotic radiosurgery is more cost effective than fixed-gantry radiosurgery. However, robotic radiosurgery has higher budget impact than fixed-gantry radiosurgery. Thus, decision-makers need to judge whether there is enough patient volume in their own setting to offset the budget impact of robotic radiosurgery.

\section{REFERENCES}

1. Pickren JW, Lopez G, Tzukada Y, et al. Brain metastases: an autopsy study. Cancer Treat Symp. 2003;2:295-313.

2. Posner JB, Chernik NL. Intracranial metastases from systemic cancer. Adv Neurol. 1978;19:579-92.

3. Andrews DW, Scott CB, Sperduto PW, et al. Whole brain radiation therapy with or without stereotactic radiosurgery boost for patients with one to three brain metastases: phase III results of the RTOG 9508 randomized trial. Lancet. 2004;363:1665-72.

4. Cqco.on.ca. Toronto: Cancer Quality Council of Ontario; c2002 [cited 2012 Dec 25]. Available from: http://www.csqi.on.ca/.

5. Boudreau R, Clark M, Nkansah E. TomoTherapy, GammaKnife and CyberKnife therapies for patients with tumours of the lung, central nervous system, or inta-abdomen: a systematic review of clinical effectiveness and cost-effectiveness. Ottawa: Canadian Agency for Drugs and Technologies in Health, 2009.

6. Wowra B, Muacevic A, Tonn J. Quality of radiosurgery for single brain metastases with respect to treatment technology: a matched-pair analysis. J Neurooncol. 2009;94:69-77.

7. Diaz-Santos NC, Amaro JAB, Cardiel GA, et al. The safety and efficacy of robotic image-guided radiosurgery system treatment for intra- and extracranial lesions: a systematic review of the literature. Radiother Oncol. 2009;89:245-53.

8. Regis J, Manabu T, Cicile G, et al. Radiosurgery with the world's first fully robotized Leskell GammaKnife PerfeXion in clinical use: a 200-patient prospective, randomized, controlled comparison with the GammaKnife 4C. Neurosurgery. 2009;64: 346-56.

9. Sharieff W, Greenspoon J, Newton T, et al. Stereotactic radiation for brain metastases with Cyberknife: The Hamilton Experience. Presented at the Radiosurgery Society Annual Scientific Meeting, San Diego, CA, February 14-15, 2013.

10. Health.gov.on.ca. Toronto: Ministry of Health and Long-Term Care; c2002. [updated 2013 February 4; cited 2012 December 15]. Available from: http://www.health.gov.on.ca/english/ providers/program/ohip/sob/physserv/physserv_mn.html.

11. Clinicaltrials.gov. Bethesda: National library of Medicine, the National Institutes of Health; c2000-02 [updated 2011 May 12; cited 2012 December 31]. Available from: http://clinical trial.gov/ct2/show/NCT01353573?term=greenspoon\&rank=1 .

12. Lal L, Byfield S, Chang E, et al. Cost-effectiveness analysis of a randomized study comparing radiosurgery with radiosurgery and whole brain radiation therapy in patients with 1 to 3 brain metastases. Am J Clin Oncol. 2012;35(1):45-50.

13. Mehta M, Noyes W, Craig B, et al. A cost-effectiveness and costutility analysis of radiosurgery vs. resection for single-brain metastases. Int J Radiat Oncol Biol Phys. 1997;39:445-54.

14. Vuong DA, Rades D, van Eck AT, Horstmann GA, Busse R. Comparing the cost-effectiveness of two brain metastasis treatment modalities from a payer's perspective: stereotactic radiosurgery versus surgical resection. Clin Neurol Neurosurg. 2013;115(3):276-84

15. Königsmaier H, de Pauli-Ferch B, Hackl A, Pendl G. The costs of radiosurgical treatment: comparison between gamma knife and linear accelerator. Acta Neurochir (Wien). 1998;140(11): 1101-10. 